\title{
Long-term surgical outcomes of retinal detachment in patients with Stickler syndrome
}

\author{
Devasis N Reddy' \\ Yoshihiro Yonekawa ${ }^{1-4}$ \\ Benjamin J Thomas ${ }^{1,2,5}$ \\ Eric D Nudleman ${ }^{6}$ \\ George A Williams ${ }^{1,2}$ \\ 'Oakland University William \\ Beaumont School of Medicine, \\ Rochester, MI, ${ }^{2}$ Associated \\ Retinal Consultants, William \\ Beaumont Hospital, Royal Oak, \\ MI, ${ }^{3}$ Massachusetts Eye and Ear \\ Infirmary, Boston, MA, ${ }^{4}$ Boston \\ Children's Hospital, Harvard Medical \\ School, Boston, MA, ${ }^{5}$ Florida Retina \\ Institute, Jacksonville, FL, ${ }^{6}$ Shiley Eye \\ Institute, University of California \\ San Diego School of Medicine, \\ San Diego, CA, USA
}

This article was published in the following Dove Press journal: Clinical Ophthalmology

16 August 2016

Number of times this article has been viewed

Purpose: The aim of the study was to present the long-term anatomical and visual outcomes of retinal detachment repair in patients with Stickler syndrome.

Patients and methods: This study is a retrospective, interventional, consecutive case series of patients with Stickler syndrome undergoing retinal reattachment surgery from 2009 to 2014 at the Associated Retinal Consultants, William Beaumont Hospital.

Results: Sixteen eyes from 13 patients were identified. Patients underwent a mean of 3.1 surgical interventions (range: 1-13) with a mean postoperative follow-up of 94 months (range: 5-313 months). Twelve eyes (75\%) developed proliferative vitreoretinopathy. Retinal reattachment was achieved in $100 \%$ of eyes, with ten eyes $(63 \%)$ requiring silicone oil tamponade at final follow-up. Mean preoperative visual acuity (VA) was 20/914, which improved to 20/796 at final follow-up $(P=0.81)$. There was a significant correlation between presenting and final VA $(P<0.001)$, and patients with poorer presenting VA were more likely to require silicone oil tamponade at final follow-up $(P=0.04)$.

Conclusion: Repair of retinal detachment in patients with Stickler syndrome often requires multiple surgeries, and visual outcomes are variable. Presenting VA is significantly predictive of long-term VA outcomes.

Keywords: pediatric retina, proliferative vitreoretinopathy, retina, scleral buckle, vitrectomy, vitreoretinal surgery

\section{Introduction}

Stickler syndrome - alternately known as hereditary arthro-ophthalmopathy - is an inherited disorder of collagen formation manifesting itself through a spectrum of ocular, orofacial, auditory, and musculoskeletal abnormalities. ${ }^{1,2}$ The disease is relatively uncommon, with a reported incidence of one in 7,500-9,000 births, and presents with highly variable signs and symptoms in affected individuals. ${ }^{3}$ This diversity of phenotypes reflects the heterogeneous mutations affecting the formation of Types II, IX, and XI collagen, which are integral to the structure and function of the vitreous, cartilage, and other connective tissues. ${ }^{4,5}$

Specific ocular manifestations include high myopia, optically empty vitreous, openangle glaucoma (from dysgenesis of the anterior chamber drainage angle), cortical cataract, and perivascular retinal lattice degeneration with strong vitreous adhesions along the borders of these lesions. ${ }^{6}$ These vitreoretinal changes predispose patients to the development of giant retinal tears (GRTs) and associated rhegmatogenous retinal detachments (RRD) relatively early in life, often in the second and third decades of life. ${ }^{4,6,7}$ Stickler syndrome is the most common inherited cause of RRD, in spite of its low incidence overall. ${ }^{8}$ 
Given the low incidence of Stickler syndrome, long-term data on surgical outcomes in patients with Stickler syndrome are few: only two published studies have previously detailed the surgical treatment and outcomes of retinal detachment (RD) repair in patients with Stickler syndrome. ${ }^{9,10} \mathrm{We}$ reviewed the long-term anatomical and visual outcomes of $\mathrm{RD}$ in our cohort of patients with Stickler syndrome who underwent surgical repair.

\section{Patients and methods}

This study is a retrospective, single-center, interventional, case series of patients with Stickler syndrome undergoing surgery for RD. Patients were screened via billing codes, surgical logs, and genetic testing and were seen during the period from January 1, 2009, through December 31, 2014. Patient records were reviewed to confirm a diagnosis of Stickler syndrome, and data collection was completed, as outlined later. Patients were excluded if they did not have a confirmed diagnosis of Stickler syndrome, if there were confounding causes for RRD (eg, ocular trauma) or confounding reasons for severe vision loss (eg, end-stage glaucoma), or if they did not undergo surgery primarily for RD. The study was approved by the Western Institutional Review Board, and it complied with the Health Insurance Portability and Accountability Act of 1996 and adhered to the tenets of the Declaration of Helsinki. The requirement to obtain informed consent from patients was waived due to the nature of the study.

Data collection included demographic information, details of surgical interventions, and multimodal retinal imaging including color fundus photography, fluorescein angiography, and optical coherence tomography. Best corrected visual acuity (BCVA) and anatomical outcomes were recorded preand postoperatively. Snellen visual acuities were converted to the logarithm of the minimum angle of resolution units for statistical analyses. Nonparametric paired significance testing was performed using the Wilcoxon signed-rank test; statistical tests were two-tailed, and significance was defined as $P<0.05$. Stata Version 9.0 (StataCorp LP, College Station, TX, USA) was used for statistical analyses.

\section{Results}

Sixteen eyes from 13 patients with Stickler syndrome met the inclusion criteria, with three patients $(23 \%)$ undergoing bilateral surgeries for RD repair. The average age at the time of initial diagnosis with RRD was 10.4 years (range: 1 month-22 years), and the mean follow-up duration was 94 months (range: 5-313 months). Table 1 summarizes the demographic data for this cohort.
Table I Demographic and clinical features of patients with Stickler syndrome undergoing RD repair

\begin{tabular}{ll}
\hline Feature & Number \\
\hline $\begin{array}{l}\text { Age ( } \mathrm{n}=13 \text { patients), years } \\
\text { Mean (median, range) }\end{array}$ & $10.4(1 \mathrm{I}, \mathrm{I}$ month-22 years) \\
$\begin{array}{l}\text { Sex } \\
\text { Male }\end{array}$ & $10(77 \%)$ \\
$\quad$ Female & $3(23 \%)$ \\
Eye ( $\mathrm{n}=16$ eyes) & $7(44 \%)$ \\
$\quad$ Right & $9(56 \%)$ \\
Left & $3(19 \%)$ \\
Type of retinal break & $5(31 \%)$ \\
$\quad$ Small single break & $8(50 \%)$ \\
$\quad$ Multiple small breaks &
\end{tabular}

Abbreviations: RD, retinal detachment; GRT, giant retinal tear.

Mean visual acuity (VA) at the time of presentation was 1.66 (SD \pm 0.86 ; Snellen equivalent: 20/914), which slightly improved at final evaluation to 1.60 ( $\mathrm{SD} \pm 1.01$; Snellen equivalent: 20/796; $P=0.81)$. Improvement in VA was seen in five eyes (31\%), while five (31\%) remained stable and six (37.5\%) experienced a decline.

Table 2 summarizes the anatomical outcomes and details of surgical intervention. Three eyes $(18.8 \%)$ had single retinal breaks, five eyes $(31.3 \%)$ had multiple breaks, and the remaining eight (50\%) had GRTs with at least 3 clock-hours

Table 2 RD repair in patients with Stickler syndrome

\begin{tabular}{ll}
\hline Feature & Number \\
\hline Number of surgeries & $3(18.8 \%)$ \\
One surgery & $13(81.2 \%)$ \\
Multiple surgeries & 3.1 (range, I-13) \\
Average number & \\
Initial surgery & $5(31 \%)$ \\
Scleral buckle only & $7(44 \%)$ \\
Vitrectomy only & $4(25 \%)$ \\
Buckle and vitrectomy & \\
Silicone oil usage & $14(88 \%)$ \\
Used at any point & $10(63 \%)$ \\
Present at last visit & \\
Anatomical outcomes & \\
Success & $16(100 \%)$ \\
Failed & $0(0 \%)$ \\
Scleral buckle success & $5(100 \%)$ \\
Vitrectomy success & $7(100 \%)$ \\
Buckle and vitrectomy success & $4(100 \%)$ \\
Functional outcomes & Average change (logMAR) \\
Scleral buckle & -0.24 \\
Vitrectomy & 0.08 \\
Buckle and vitrectomy & -0.05 \\
\hline
\end{tabular}

Note: anitial surgery type.

Abbreviations: RD, retinal detachment; logMAR, logarithm of the minimum angle of resolution. 
of peripheral retinal involvement. The mean size of the GRTs was 3.25 clock-hours. Proliferative vitreoretinopathy developed at any point in 12 eyes $(75 \%)$. The mean number of vitreoretinal surgeries required for retinal reattachment was 3.1 (range: 1-13). In eyes requiring multiple surgical interventions (13 of 16 eyes, $81.3 \%$ ), the mean interval prior to redetachment (after the primary intervention) was 4.8 months (range: 4 days-36 months). All retinas were completely reattached at the final visit. Six retinas $(37 \%)$ were attached without silicone oil tamponade, while the remaining ten retinas $(63 \%)$ were attached utilizing silicone oil tamponade. There were no eyes with hypotony.

\section{Discussion}

Stickler syndrome, though rare, presents certain diagnostic and therapeutic challenges to the vitreoretinal surgeon. Several factors may contribute to the complexity of RRD repair in these patients, including the presence of multiple retinal tears, GRTs, the young age of presentation, and the abnormal vitreous syneresis seen in patients with Stickler syndrome. This difficulty is evident in the average number of surgeries until complete RRD repair in these patients, which was shown to be 3.1 in our study. Given these confounding factors, however, all 16 eyes in our study achieved successful retinal reattachment, with a mean postoperative follow-up to almost 8 years. In our series, patients with poorer presenting VA were more likely to require silicone oil tamponade for successful reattachment. Anatomical success in the presence of silicone oil tamponade is consistent with previous studies, which also showed anatomical success when silicone oil tamponade was utilized..$^{9,10}$

In spite of successful anatomical outcomes, visual outcomes remain modest, improving only slightly from VA at presentation: in our cohort, patients with RRD presented with an average best corrected visual acuity of 20/914 (Snellen equivalent). After an average of 3.1 surgeries, mean best corrected visual acuity only improved to 20/796 - though this improvement did not achieve statistical significance $(P=0.81)$ - and the VA outcomes were widely variable, ranging from $20 / 20$ to hand motions. Of note, presenting VA bore prognostic significance, as it showed strong correlation with the postoperative VA at final follow-up $(P<0.001)$. The variability of VA outcomes after surgical intervention for $\mathrm{RD}$ in our cohort is similar to those VA results reported by Abeysiri et al, ${ }^{9}$ where $33 \%$ of patients had improved VA, $40 \%$ maintained VA, and 27\% experienced a decrease in VA, and Alshahrani et al, ${ }^{10}$ where $64.29 \%$ of patients had improved VA while $28.57 \%$ maintained VA.
Most eyes $(81.3 \%)$ in our study required multiple surgeries to successfully achieve retinal reattachment. The five eyes demonstrating the greatest improvements in VA postoperatively required, on average, approximately five surgeries before retinal reattachment.

When surgical treatments are performed to repair these difficult and complex RDs, our series demonstrated that initial surgery with scleral buckle, vitrectomy, and combined vitrectomy and scleral buckle procedures all yielded equally successful anatomical outcomes with $100 \%$ retinal reattachment. These results contrast to those of previous studies, one of which showed superior anatomical outcomes with vitrectomy, while the other suggested combined vitrectomy and scleral buckle procedure with silicone oil injection to be the treatment of choice for treating RD in patients with Stickler syndrome. ${ }^{9,10}$

Our data overall underscore a few critical points for Stickler syndrome patients with RD: first, the $75 \%$ rate of proliferative vitreoretinopathy seen in our study, as well as the average of 3.1 surgeries required to repair these RDs, highlights the complicated nature of surgical therapy for these patients. Second, in our cohort, patients presented with RRD at a mean age of 10.5-years old, which is a younger age compared to previous series. ${ }^{9,10}$ Finally, given this younger age of presentation, as well as the challenges of surgery and the modest visual gains, these data further reinforce the importance of early diagnosis and prophylactic therapy in patients with Stickler syndrome. Previous studies have demonstrated that prophylactic cryotherapy markedly reduces the risk of RDs in Stickler syndrome patients. ${ }^{11,12}$ Recognition of patients with a Stickler ocular phenotype by primary care doctors, ophthalmologists, and other health care providers is essential for preventing the severe retinal complications in these patients.

Limitations of our study include its retrospective design and the small sample size studied - a frequent limitation of reviews of Stickler syndrome. This small sample size inevitably magnifies any diagnostic or therapeutic biases in our cohort, and significant caution is warranted in drawing conclusions from subgroup analyses. However, as our data tended to corroborate those of previous publications, the conclusions drawn from the overall cohort are likely to be sound.

\section{Conclusion}

Our study affirms the feasibility of successful retinal reattachment in cases of RRD in Stickler syndrome, usually after multiple vitreoretinal surgeries. However, VA outcomes are highly variable and show only a small improvement from 
presenting VA in long-term follow-up overall. Complicated peripheral retinal pathology, high rates of GRT, high rates of proliferative vitreoretinopathy, and frequent redetachment requiring additional surgeries are the norm in this cohort, and early diagnosis (within the first decade of life) should be the goal for these patients.

\section{Disclosure}

George A Williams is a consultant for Alcon Surgical. The other authors report no conflicts of interest in this work.

\section{References}

1. Snead MP, Yates JR. Clinical and molecular genetics of Stickler syndrome. J Med Genet. 1999;36(5):353-359.

2. Stickler GB, Hughes W, Houchin P. Clinical features of hereditary progressive arthro-ophthalmopathy (Stickler syndrome): a survey. Genet Med. 2001;3(3):192-196.

3. Stickler Syndrome [webpage on the Internet]. Genetics Home Reference [updated 2013]. Available from: http://ghr.nlm.nih.gov/condition/ stickler-syndrome. Accessed February 23, 2013.

4. Snead MP, Mcninch AM, Poulson AV, et al. Stickler syndrome, ocular-only variants and a key diagnostic role for the ophthalmologist. Eye (Lond). 2011;25(11):1389-1400.
5. Robin NH, Moran RT, Ala-Kokko L [webpage on the Internet]. Stickler Syndrome; 2000 [updated November 26, 2014]. In: Pagon RA, Adam MP, Ardinger HH, et al, editors. GeneReviews [Internet]. Seattle, WA: University of Washington; 1993-2015. Available from: http:// www.ncbi.nlm.nih.gov/books/NBK1302/. Accessed July 16, 2016.

6. Parma ES, Korkko J, Hagler WS, Ala-Kokko L. Radial perivascular retinal degeneration: a key to the clinical diagnosis of an ocular variant of stickler syndrome with minimal or no systemic manifestations. Am J Ophthalmol. 2002;134(5):728-734.

7. Shuler M, Sullivan J, Hurley B, McNamara J. Vitreoretinal dystrophies. Pediatric Retina. 2011 ed. London: Springer Berlin Heidelberg; 2011:315-319. [Kresge Reference Library. Web. 23 May 2015].

8. Richards AJ, Scott JD, Snead MP. Molecular genetics of rhegmatogenous retinal detachment. Eye (Lond). 2002;16(4):388-392.

9. Abeysiri P, Bunce C, da Cruz L. Outcomes of surgery for retinal detachment in patients with stickler syndrome: a comparison of two sequential 20-year cohorts. Graefes Arch Clin Exp Ophthalmol. 2007;245(11): 1633-1638.

10. Alshahrani ST, Ghazi NG, Al-Rashaed S. Rhegmatogenous retinal detachments associated to Stickler syndrome in a tertiary eye care center in Saudi Arabia. Clin Ophthalmol. 2016;10:1-6.

11. Ang A, Poulson AV, Goodburn SF, Richards AJ, Scott JD, Snead MP. Retinal detachment and prophylaxis in type 1 stickler syndrome. Ophthalmology. 2008;115(1):164-168.

12. Fincham GS, Pasea L, Carroll C, et al. Prevention of retinal detachment in stickler syndrome: the Cambridge prophylactic cryotherapy protocol. Ophthalmology. 2014;121(8):1588-1597.
Clinical Ophthalmology

\section{Publish your work in this journal}

Clinical Ophthalmology is an international, peer-reviewed journal covering all subspecialties within ophthalmology. Key topics include: Optometry; Visual science; Pharmacology and drug therapy in eye diseases; Basic Sciences; Primary and Secondary eye care; Patient Safety and Quality of Care Improvements. This journal is indexed on

Submit your manuscript here: http://www.dovepress.com/clinical-ophthalmology-journal

\section{Dovepress}

PubMed Central and CAS, and is the official journal of The Society of Clinical Ophthalmology (SCO). The manuscript management system is completely online and includes a very quick and fair peer-review system, which is all easy to use. Visit http://www.dovepress.com/ testimonials.php to read real quotes from published authors. 